\title{
Saliva Stimulants and the ORal Health of Geriatric Patients
}

\author{
K.K. MÄKINEN* \\ D. Pemberton ${ }^{1}$ \\ J. Cole ${ }^{1}$ \\ P.-L. MÄKINEN \\ C.-Y. Chen \\ P. LAMBERT ${ }^{1}$
}

Department of Biologic and Materials Sciences

School of Dentistry

University of Michigan

Ann Arbor, Michigan 48109-1078

'Veterans Administration Medical Center

Dayton, Ohio 45428

"Corresponding author

Adv Dent Res 9(2):125-126, July, 1995 oot-surface caries (RSC) has been recognized as a specific and important dental disease. Significant advances have been made in the pathology and microbiology of RSC, and the need to standardize the guidelines for recording RSC data has been recognized. Researchers have emphasized the increasing impact RSC will have on the geriatric population, especially since the methods to treat and prevent this disease are limited. The purpose of this study was to investigate the possibility of limiting RSC in a Veterans Administration (VA) patient population, using polyol-containing saliva stimulants that were voluntarily consumed by residents of a VA Medical Center (VAMC) over a period of from six to 30 months. Another aim was to study the effect of this program on the gingival health of periodontal patients.

Key words: Root surface caries, gingival health, sugar substitutes, xylitol, sorbitol.

Presented at the 4th World Congress on Preventive Dentistry, "Trends in Prevention-Promotion of Oral Health within General Health Care...Possibilities and Limitations in Preventive Dentistry", September 3-5, 1993, University of Umeå, Sweden, co-sponsored by the World Health Organization and the International Association for Dental Research

\section{METHODS}

This study was carried out at the VAMC in Dayton, Ohio, USA, in domiciliary patients who consented to the program and completed a minimum of six months of participation. These subjects constituted a skewed human sample as a result of underlying diseases, smoking, abuse of alcohol and drugs, poor oral hygiene, and other aberrations. The subjects were randomly assigned at baseline to either an $\mathrm{X}$ $(n \approx 40)$ or an $S(n \approx 40)$ group. Those VAMC patients who did not participate in the study $(\mathrm{n} \approx 100)$ served as controls (C).

The numbers of subjects examined at six-month recalls during the program and during pre-study years are shown in the Table.

The clinical examiner and the subjects were blinded with regard to the bulk sweetener (xylitol or sorbitol) present in the saliva stimulants. The subjects used either dragées or gums (the subjects chose whether to use gum or dragées; $90 \%$ preferred dragées). The dragées were coated tablet-like confections that consisted almost entirely of xylitol or sorbitol. Both the gum and the dragées were made to contain either xylitol or sorbitol. A dental assistant carried out the delivery of the saliva stimulants and monitored their use. A sufficient quantity of product was given to each patient for about one month's consumption. A logbook was kept to monitor the deliveries and observations on compliance. The subjects were instructed to consume from three to five dragées at a time five times a day, or to chew one stick of gum five times a day, after main meals and sugary snacks. The amount of $S$ and $X$ present in a daily "dose" was up to $10.7 \mathrm{~g}$. Only active supragingival root-surface caries (SGRSC) lesions were included. According to standard VAMC practice, all SGRSC lesions observed at the six-month examinations were removed, treated with $\mathrm{SnF}_{2}$, and restored with amalgam or light-cured glass ionomer. Very small lesions were not restored, but were cleaned off and treated with $\mathrm{SnF}_{2}$. The significance levels of differences between the numbers of SGRSC lesions were preliminarily calculated by means of ANOVA (a detailed report will be published separately). The individual patient-group data were used to establish the baseline for each group. The results should be viewed against the low number of subjects available after two years.

The subjects in the periodontal study consisted of outpatients visiting the VAMC every sixth month. The clinical products tested and the general study protocol were as above. There were 29,28 , and 30 subjects in the X, S, and $\mathrm{C}$ groups, respectively. The following measurements will be reported here: Plaque Index (Silness and Löe, 1964) and Gingival Index (Löe and Silness, 1963). 
TABLE

THE NUMBER OF SUBJECTS EXAMINED DURING THE PROGRAM

\begin{tabular}{|c|c|c|c|c|c|c|c|c|c|c|}
\hline \multirow{3}{*}{$\begin{array}{l}\text { Years: } \\
\text { Group }\end{array}$} & \multirow{3}{*}{$-2 *$} & \multirow{3}{*}{-1.5} & \multirow{3}{*}{-1.0} & \multirow{3}{*}{-0.5} & \multirow{3}{*}{0} & \multirow{3}{*}{0.5} & \multirow{3}{*}{1.0} & \multicolumn{3}{|c|}{ Use of Stimulants } \\
\hline & & & & & & & & 1.5 & 2.0 & 2.5 \\
\hline & & & & & & & & & & \\
\hline $\mathrm{X}$ & 17 & 19 & 22 & 23 & 41 & 41 & 35 & 25 & 19 & 13 \\
\hline$S$ & - & 17 & 20 & 20 & 42 & 42 & 36 & 26 & 19 & 15 \\
\hline C & 65 & 69 & 70 & 84 & $\begin{array}{c}106 \\
\uparrow \\
\mathrm{Ba}\end{array}$ & 106 & 103 & $\begin{array}{r}87 \\
\\
\text { ars) }\end{array}$ & 70 & 53 \\
\hline
\end{tabular}

*For several subjects, supragingival root-surface caries information from pre-study years $(-0.5$ to -2$)$ was available.

\section{RESULTS}

The mean number of SGRSC lesions in the combined patient population was 100 before treatment (per 1000 teeth with exposed roots). The rate of SGRSC in the $C$ group stayed at this level after baseline. In the treated groups, the rate of SGRSC dropped significantly $(p<0.05)$ after the first period (six months) and stayed significantly lower than in the $\mathrm{C}$ group during the rest of the observation period. The rate of SGRSC was lower in the $X$ group than in the S group, the difference between groups approaching significance $(p=$ 0.06 ). The saliva levels of mutans streptococci and lactobacilli tended to be lower, but not significantly, in the $X$ group compared with the $S$ group.

The mean Gingival Index scores decreased significantly $(\mathrm{p}$ $<0.05$ ) by the end of the second year of treatment in both polyol groups, which did not differ. The mean Plaque Index scores were reduced during treatment, but not significantly. The $\mathrm{X}$ and $\mathrm{S}$ groups did not differ.

\section{CONCLUSIONS}

All subjects who completed the program were satisfied with the long-term use of saliva stimulants, partly because voluntarism of participation was emphasized. For several subjects, the discontinuation of the program was a disappointment. Patients who began the program but were not motivated enough dropped out soon after its beginning. Several compliant subjects dropped out because they were discharged from the VAMC. The consumption of both polyols was associated with reduced incidence of SGRSC. The use of $\mathrm{X}$ was associated with almost no recurrence of SGRSC, but the small number of subjects does not support strong conclusions. The periodontal outpatient group showed improved oral hygiene as determined by means of plaque and gingival indices. The results were explained in terms of increased daily salivary gland output stimulated by frequent use of chewable and suckable products that contained a slowly fermentable or an almost-non-fermentable carbohydrate sweetener.

These results suggest that the addition of a dragée (or a lozenge or a gum) program to the preventive strategies used at VAMCs may provide additional protection against SGRSC and may improve the resident patients' oral hygiene.

\section{REFERENCES}

Löe H, Silness J (1963). Periodontal disease in pregnancy. I. Prevalence and severity. Acta Odontol Scand 21:533-551.

Silness J, Löe H (1964). Periodontal disease in pregnancy. II. Correlation between oral hygiene and periodontal condition. Acta Odontol Scand 22:121-135. 